Der Sonntag galt dann der Glattalp. Mit der freundlicherweise zur Verfügung gestellten Werkseilbahn gelangten die Exkursionsteilnehmer am frühen Morgen vom innern Bisistal bei der Sahlialp rasch und bequem über $700 \mathrm{~m}$ Höhendifferenz in die verkarstete Hochmulde, in welcher der zur Zeit durch einen künstlichen Stollen größ_ tenteils entleerte Glattalpsee eingebettet liegt. Schon auf der letzten Fahrtstrecke erhielt man, in geringer Distanz vom Boden, prächtigen Einblick in die Reihen von Dolinen und Schlucklöchern, die sich in der Regel an Klüfte und Verwerfungslinien halten und dem Wasser das Eindringen durch die anstehenden Zementsteinschichten in den liegenden, der Verkarstung besonders Vorschub leistenden Quintnerkalk ermöglichen. Die im Zusammenhang mit dem Kraftwerkbau unternommenen Arbeiten haben zur Freilegung einer großen $\mathrm{Zahl}$ solcher Erscheinungen geführt und gestatteten das nähere Studium ihrer Anlage; soweit Gefahr besteht, daß sie die Aufstauung des Sees beeinträchtigen würden, müssen sie mit Beton abgedichtet werden - ein Verfahren, das sich, zumal keine hohe Staumauer errichtet werden muß, sehr eindrücklich von der Gestaltung anderer, bekannterer Stauräume im Hochgebirge unterscheidet. Der hervorragende Anschauungsunterricht, der sich einem rings um den See in dieser Beziehung bot, wurde, vor allem am Nachmittag, ergänzt durch die Untersuchung all der verschiedenen Karrenformen, die in seltener Vollständigkeit, gewissermaßen als Schulbeispiele, sich hier vorfinden, und deren Entstehung und Entwicklung Dr. Bögli sehr anschaulich, nun am Objekt selber erläuterte. Zahlreiche weitere Erscheinungen Gletscherschliffe, Deltabildung, Bergstürze, Sackungen usw. präsentieren sich auf der Glattalp ihrerseits in prächtigen Zeugnissen. Die Exkursion, die in der Mittagspause durch eine kurze Darlegung des in Ausführung begriffenen Kraftwerksystems noch ergänzt wurde, erlaubte damit sehr vielesitige Einsicht und erfüllte aufs Schönste die in sie gesetzten Erwartungen.

\title{
DER ATLAS VON NIEDERÖSTERREICH
}

Im Verein für Landeskunde von Niederösterreich und Wien wurde 1925 zum ersten Male der Plan entwickelt, einen umfassenden landeskundlichen Atlas Niederösterreichs und Wiens zu schaffen. Aus finanziellen Gründen mußte die Idee dann fallen gelassen werden. Prof. Dr. H. HAssinger, griff sie im Jahre 1931 erneut auf, doch ließ sie sich erst 1940 in die Tat umsetzen. In den Wirren von 1945 ging das gesammelte Material aber größtenteils verloren. Erst 1949 gelang es Hassinger weitere Mittel flüssig zu machen, um das in Bruchstücken vorhandene Werk fortzusetzen. Im Mai 1951 erlebte der Betreuer noch das Erscheinen der ersten Doppellieferung. Sein Nachfolger Prof. Dr. J. Keıl führte die Arbeiten im Geiste des Begründers weiter. Seit dem Mai 1959 liegt es nun in 7 Doppellieferungen fertig vor, während der Textband noch aussteht (vgl. in Heft Geographica Helvetica IX, 1954, S. 109). Der Atlas umfaßt 127 Kartenblätter mit 238 Haupt- und Nebenkarten in Mehrfarbendruck, 1 erdgeschichtliche Übersichtstafel, 1 Bodenprofiltafel, 6 Farbtafeln mit 5o Kolorlandschaftstypenbildern, 11 Verzeichnissen, zahlreiche textliche Erläuterungen, 185 Abbildungen ur- und frühgeschichtlicher Fundbeispiele, sowie zahlreiche andere Abbildungen und graphische Darstellungen. Die Hauptkarten haben für Niederösterreich durchwegs den Maßstab 1:500 000, die Übersichtskarten meist 1:1000 ooo und die für Wien 1:66 000. Auf jeder Karte sind neben der Legende auch die Arbeitsgrundlagen angegeben, so daß das Werk zugleich eine landeskundliche Bibliographie von Niederösterreich darstellt.

Das Inhaltsverzeichnis zeigt die Fülle des gebotenen Stoffes, der ein umfassendes Bild Niederösterreichs vermittelt, wobei alle denkbaren Aspekte der Landschaft in gründlichster Weise wiedergegeben sind. Die Hauptkapitel heißen: Niederösterreich im Mitteleuropäischen Raum, die Karten zeigen historische, geologische, klimatische und vegetationsmäßige Lagebeziehungen; Topographie, Politisch-Administrative Eintcilung; Bau und Oberflächenformen neben den üblichen Karteninhalten findet sich hier eine spezielle über die verkarstungsfähigen Gesteine und Höhlen - Klima und Phänologic - in dieser Serie sind zahlreiche Spezialkarten vorhanden, die den Botaniker und Zoologen zu interessieren vermögen wie etwa der Beginn der Kirschblüte, das Stecken der Kartoffeln, das Ende des Schwalbenzuges u. a. m. - Bodenvorhältnisse, Vegetation und Tierwelt, Landschaftsschutzgebiete - auch hier liegen zahlreiche Spezialkarten vor, so etwa die Leitlinien des Vogelzuges und der Distelfalterwanderflüge oder Verbreitung von Ziesel und Hamster und Massenvermehrung (1881-1944) einiger Waldschädlinge 
- Landesgeschichte, Siedlungen und Siedlungsräume früher und heute - dieses Kapitel ist sehr eingehend behandelt und enthält auch zahlreiche kartographische Ausschnitte und Hinweise auf ältere Darstellungen Niederösterreichs - Dic Bevölkerung - für den Sprachforscher bestehen aufschlußreiche Angaben über den Aufbau und Verfall der niederösterreichischen Dialektlandschaft - Land-und Forstwirtschaft; Industrie und Gewerbe; Entwicklung des Verkehrsnetzes, Handel und Fremdenverkchr; zentrale Einrichtungen, Zentrale Orte, Sozialwirtschaftliche Struktur; Landschaftsgliederung; Landschaftstypen (Farbaufnahmen typischer Gebiete Niederüsterreichs durch E. Arnberger), Landschaftsschutzgebicte und schließlich als letztes Kapitel Volkskunde.

Der Atlas gibt somit nicht nur dem Geographen, welcher Richtung er auch angehöre, für seine Studien weitgehende Auskunft, sondern auch der Historiker, der Wirtschaftswissenschafter und Landesplaner wird darin Fundgruben finden. Aus dem Vergleich einzelner Karten läßt sich das Reifen und der zeitweise Zerfall von Kulturlandschaften verfolgen. Der ganze Niederösterreichische Raum steht in engen Wechselbeziehungen zur Großstadt Wien. Sie konnte deshalb nicht aus der Betrachtung ausgeklammert werden. Daher sind mehrere Haupt- und Nebenkarten dem Bundesland Wien gewidmet. Der Atlas von Niederösterreich ist als ein grundlegendes Werk zur Landeskunde zu werten, das unzählige Anregungen zu vermitteln und als Beispiel zu dienen vermag.

Der Preis des Gesamtwerkes, das unter der redaktionellen Leitung Dr. Erik Arnbergers entstanden und in der Kartographischen Anstalt Freytag-Berndt und Artaria in Wien verlegt worden ist, beträgt im Buchhandel für die Loseblattausgabe (Format $58 \times 46 \mathrm{~cm}$ ) $\mathrm{sFr}$. 167.-, für Halbleinband mit Schraubverschluß (Format $59 \times 47 \mathrm{~cm}$ ) sFr. 202.-, Halblederband mit Schraubverschluß sFr.238.-, Halblederausgabe in Halbformat (gefalzte Blätter auf Fälze gelegt) sFr. 268.-

P. KÖCHLI

\section{PROF. DR. WALTER WIRTH 7o JAHRE ALT}

Am 23. Dezember 1961 vollendet WALTER WIRTH in Winterthur in voller Rüstigkeit sein 7o. Lebensjahr. Häufig wandert er noch mit seinen Freunden; gerne empfängt er sie in seinem von der Gattin liebevoll gepflegten Blumengarten.

Unermüdlich für sein Fach und die Kollegengemeinschaft tätig, war WIRTH einer unserer erfolgreichsten Schulgeographen, in dem Sinne, daß er überall, wo er wirkte, durch seine maßvoll-kluge und zugleich gewissenhafte Art für die Geographie Ehre einlegte. Äußere Marksteine in seinem Wirken als erster Fachlehrer für Geographie an der Kantonsschule Winterthur (seit 1921) sind die Ausarbeitung der Lehrpläne und Entwicklung der Methoden für den Oberstufenunterricht für die nach dem eidgenössischen Reglement von 1925 zum Maturitätsfach erhobene Geographie; Einrichtung und Bezug der Sammlung im Neubau der Kantonsschule 1928; Schülerübungen nach dem Reformplan des Gymnasiums 1950, die er nach eigenen und nellartigen Plänen durchführte und über die er in den Geographica Helvetica 1951/1 und 1955/2 sowie in der Geographischen Rundschau 1953/8 referiert hat. Bei den Übungen ging es ihm darum, die Schüler selbst an die Quellen geographischer Untersuchung heranzuführen.

Als 1932 der Mittelschulatlas in erweitertem Umfang erschien, hatte WIRTH am Inhaltsplan entscheidend mitgewirkt; den in Regionalkarten dargestellten Typenlandschaften der Schweiz und Europas hat er sich im Unterricht stets mit Überzeugung zugewandt. Um sie genau kennen zu lernen, beteiligte er sich an zahlreichen Exkursionen und Auslandreisen. Es ist ein Genuß, ihn aus der stets noch frischen Erinnerung erzählen zu hören, sei's von den Wanderungen auf der Kurischen Nehrung, sei's aus Böhmen, wo die Schweizergruppe an einem Tag bei den Sudetendeutschen, am andern bei den Tschechen herzlich gefeiert wurde.

Stets bedauerte er, während seiner durch lange Aktivdienstzeiten unterbrochenen Studienjahre die Grenzen der Schweiz nicht verlassen zu können. Daher ließ er sich während des Schuljahres 1929/3o beurlauben, um sich im Sommersemester in Berlin (Vorlesungen bei KREBS und Rühl), im Wintersemester in Paris (Demartonne, Demangeon) zu immatrikulieren; mit Begeisterung sprach er immer von diesen Auslandsemestern.

$\mathrm{Da} \beta$ WIRTH vor allem der Länderkunde verbunden ist, beweisen seine Untersuchungen, die er meist gemeinsam mit seinem 1942 verstorbenen Freund NIKLAUS FORRER publizierte, jeweils nach längeren Aufenthalten in den Gebieten: Juf Avers (Schweizer Geograph 1925), Insel Houat in der Bretagne (Mitteilungen der Geographisch-Ethnographischen Gesellschaft Zürich 1925/26), ein Vierländer Bauernhof (ebenda 1928/29), Kulturgeographie der Provence (Schweizer Geograph 1932). In zahlreichen Vorträgen und Exkursionen, meistens im Schoße der Geographisch-Ethnographischen Gesellschaft Zürich und der Naturwissenschaftlichen Winterthur, trat er vor die Öffentlichkeit. Im Schweizer Geograph 1939 beschrieb er drei Exkursionsrouten im Kanton Zürich und legte damit bereits den Grund zu unserem Exkursionsführer.

Nach seinem 6o. Geburtstag legte WIRTH, der jahrzehntelang keinen Vortrag, keine Exkursion und keine Sitzung der Geographielehrer ohne Not versäumt hatte, ganz allmählich und vertrauensvoll die Hefte in jüngere Hände. Rat und Auskunft aus seiner reichen, durch Lektüre 